\title{
Multi-hop Routing Protocol Design Based on Cluster Density
}

\author{
Zhi-Jun Liu ${ }^{1, a}$ and Xue-Feng Pan ${ }^{2, b}$ \\ ${ }^{1}$ College of Information Science and Engineering, WuChang ShouYi University, \\ Hubei Wuhan, China \\ ${ }^{2}$ College of Computer Science, Wuhan Qing Chuan University, \\ Hubei Wuhan, China \\ a junely8018@163.com, ${ }^{b}$ panxuefeng800103@126.com
}

\begin{abstract}
In wireless sensor network protocol, a multi hop routing protocol based on density clustering is proposed to deal with the problem of large communication overhead, unbalanced node consumption and hierarchical routing. Protocol divide clusters by density based method, and transfer the data with the base station using a multi hop approach. It can effectively balance the energy consumption of the nodes, and also effectively manage and improve the inter cluster routing. Through simulation experiments, the results show that the improved clustering multi hop routing protocol can balance the cluster head energy, improve the accuracy of data transfer, and effectively extend the life cycle of the network.
\end{abstract}

Keywords: Wireless; Sensor; Networks; Density; Multi-hop routing.

\section{Introduction}

The energy efficient of the wireless sensor networks refers to the network under the conditions of limited energy to handle the number of requests, energy efficiency is an important performance metrics of wireless sensor networks.

LEACH protocol is the first to propose clustering routing protocol of wireless sensor networks [1-3]. LEACH algorithm can guarantee the probability of each node as a cluster head node in the network, making nodes relatively balanced energy consumption. LEACH protocol using data compression technology and dynamic clustering routing technology in the processing of data highly relevant[4], since the data integration efforts, a lot of redundant data is eliminated, better performance in terms of energy consumption, improve network scalability and robustness. 


\section{The Basic Idea of the Based Density Clustering Multi-hop Routing Protocol}

In the clustering routing algorithm, the energy consumption of cluster heads to consumption than the non-cluster head nodes, cluster head nodes to responsible and cluster all member nodes of a communication, and a long-distance communication with the base station, in addition to the cluster head node also completed data fusion. To avoid a node too quickly consume energy, LEACH algorithm uses random cluster head rotation method [5], the energy consumption of the cluster head in equal shares to all nodes, but clusters formed and likely to cause uneven distribution of each node in the cluster load imbalance, caused a part of the node energy consumption is too fast to reduce the impact of the entire sensor network life cycle.

For LEACH algorithm cluster head selection algorithm, establish and data transmission clusters made improvements, the main objective to improvements to reduce system power consumption and prolong the network life cycle, extended LEACH scope. The core of based density cluster multi-hop routing protocol is the energy balance of static clustering multi-hop routing algorithm. The basic ideas of algorithm design are:

(1) Dividing the fixed clusters class regional by density-based, form a optimization cluster class structure, communication between nodes is limited in the local area, effectively reducing automatic clustering stage energy costs.

(2) In the cluster class regional by dynamically select cluster head, making each node in the cluster shared equally traffic, avoiding the rapid failure of a single node. Select the cluster head node based on the current energy value, expanding the scope of LEACH.

(3) In the form of a plurality of routes for load balancing cluster each class region as a whole, single-hop head way to communicate with the cluster, reducing the overall network transmission delays, saving energy.

\section{Design of Improvement Agreement}

\subsection{Density based fixed cluster type region partition}

LEACH algorithm uses self-organizing, localized method to divide the class clusters, cluster formation is completely random, is not involved in the BS(Based Station), while clusters of class structure is not fixed, each round must reorganize clusters class. However, due to the formation of clusters of classes are random and can not be guaranteed in each round can be the most optimal cluster partitioning scheme. At the same time, each round must reorganize clusters class, cluster head large overhead. Improvement Protocol clusters divided by the 
density of the region, and cluster once formed, the cluster area is fixed, effectively reduce the self-organization of each round cluster of energy consumption. The definition of based the density partition:

Definition 1: Set the region $\varepsilon$ - neighborhood: a given subject area within a radius of $\varepsilon$.

Definition 2: If the $\varepsilon$ - neighborhood of an object that contains at least a minimum number MinPts objects, the object is called the core object.

Definition 3: Given a set of objects D, if p is the $\varepsilon$ - neighborhood q, and $q$ is a core object, the object p q departing from the object is directly density reachable.

Definition 4: If there is a chain of objects p1, p2, .., pn, p1 =q, pn = p, for pi $\in \mathrm{D},(1<=\mathrm{i}<=\mathrm{n}), \mathrm{pi}+1$ is from about pi and $\varepsilon$ direct MitPts density reachable, the object is from the object $\mathrm{p}$ and $\mathrm{q}$ on $\varepsilon$ MinPts density reachable.

Definition 5: If in objects collection D exists an object $\mathrm{o}$, making the object $\mathrm{p}$ and $\mathrm{q}$ is from o about $\varepsilon$ and MinPts density reachable, then the object $\mathrm{p}$ and $\mathrm{q}$ is about $\varepsilon$ and density MinPts connected.

Calculating the distance between the wireless sensor nodes by means of Minkowski distance metrics obtained, compare distance and node object $\varepsilon$ neighborhood to determine the relationship of density. Suppose $\mathrm{x}$ and $\mathrm{y}$ are corresponding features, $\mathrm{n}$ is the dimension of features. $\mathrm{x}$ and $\mathrm{y}$ Minkowski distance measure[6] form is:

$$
d(x, y)=\left[\sum_{i=1}^{n}\left|x_{i}-y_{i}\right|^{r}\right]^{1 / r}
$$

Through checking the wireless sensor networks each node object $\varepsilon$ neighborhood to find zoning. If a node $\mathrm{p}$ of $\varepsilon$ - neighborhood contains more than MinPts objects, create a core object $\mathrm{p}$ as a new cluster. Then, DBSCAN repeatedly seek from these core objects directly density-reachable objects, this process may involve some density up clusters merge. When no new points can be added to any cluster, the process ends.

The clustering algorithm description: Input: containing $\mathrm{n}$ objects database, radius $\varepsilon$, the minimum number MinPts. Output: all generated clusters to achieve the density requirements.

(1) REPEAT

(2) Untreated extract a point from the database;

(3) IF extraction point is the core point THEN identifies all points density-reachable from the object to form a cluster;

(4) ELSE point is extracted edge points (non-core subject), out of this cycle, and look for the next point;

(5) UNTIL all points have been processed; 
Different with LEACH algorithms, cluster class area to be divided by the BS uses a centralized approach. The number of clusters class area divided by the radius of the $\varepsilon$ value is determined, and the value of the radius $\varepsilon$ network system according to a pre-specified class $\mathrm{H}$ to determine the optimal cluster. The area sensor network coverage accordance with the density distribution rules are divided into clusters $\mathrm{H}$ class area by BS, nodes within each region is relatively concentrated, shown in Fig 1.

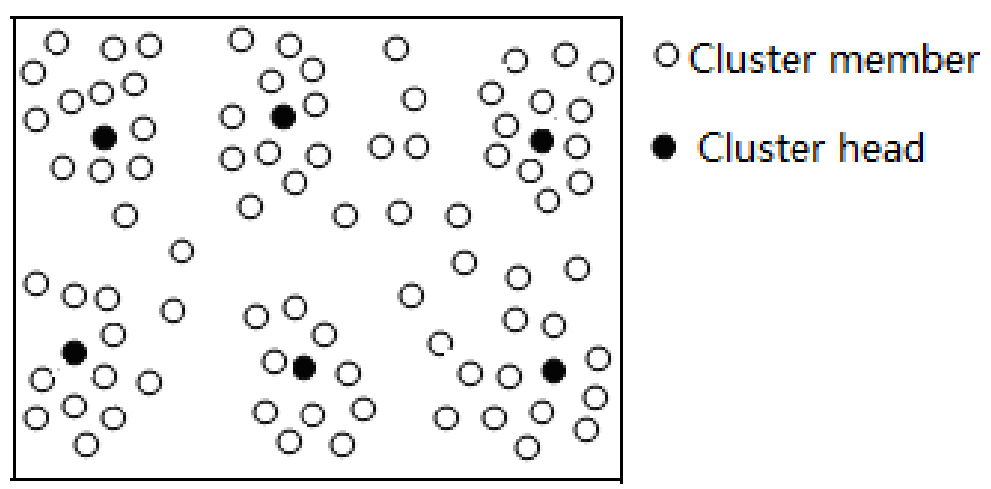

Fig.1. Cluster class region division

Class clusters region divided is performed in the first round, once division is complete, the entire network life cycle will not change. For this reason, in the next period of time (from the beginning of the second round), the algorithm is fully distributed, according to the local node complete information to determine its status, no longer need any control information BS. This can greatly reduce the amount of communication with the BS initialization phase, shortening the time of clusters, reducing the delay of the network, increasing the effective working time of the sensor.

The purpose of density-based clustering calculation cluster head number his such that the minimum total energy consumption of the network in each round. Assuming $\mathrm{N}$ sensor nodes are randomly distributed in a square area $\mathrm{M} * \mathrm{M}$, and the sensing area is divided into clusters class $\mathrm{H}$ region. Wireless energy model and wireless channel models, each round and each cluster head consumes energy, including sending broadcast packets, receiving packets other nodes, packet data fusion, data processing after sending a few packets parts [7], then there is the following energy formula:

$E_{\text {head }}=k *\left(E_{\text {elec }}+\varepsilon_{\text {amp }} * d_{\text {broadcast }}^{2}\right)+\left(\frac{N}{H}-1\right) * K^{*} E_{\text {elec }}+E_{\text {aggr }}+k *\left(E_{\text {elec }}+\varepsilon_{\text {amp }} * d_{\text {toBS }}{ }^{2}\right)$ 
$d_{\text {toBS }}$ is a cluster head to the BS distance, $d_{\text {broadcast }}$ is cluster head sent from the broadcast, due to cluster class area has been divided and fixed cluster head sends a broadcast range will be confined within the cluster class area. Thus, it can be converted into a linear representation associated with $\mathrm{H}$. The non-cluster head node energy consumption consists of two parts: a broadcast data receiving cluster head package, another is to send its own packets, the formula can be written as follows

$$
E_{\text {non_head }}=k * E_{\text {elec }}+k *\left(E_{\text {elec }}+\varepsilon_{\text {amp }} * d_{\text {tohead }}^{2}\right)
$$

$d_{\text {tohead }}$ is the distance between each non-cluster head node and cluster head. The conversion formula 1-3 and after removal of the H-independent constant, the number of problems within the cluster is converted into a mathematical problem on function extreme value:

$$
E_{\text {total }}(H)=k *\left(2 * E_{\text {elec }}+\varepsilon_{\text {amp }} * d_{\text {toBS }}^{2}\right) * H+N * k * \varepsilon_{\text {amp }} * \frac{M^{2}}{2 \pi} * \frac{1}{H}+T
$$

For $E_{\text {total }}(H)$ first-order derivative, so that it is obtained after the 0 that is directly proportional area M2 optimal cluster head number $\mathrm{H}$ and the total number of sensor network node $\mathrm{N}$ and the sensing area, the distance is inversely proportional relationship with the BS. So we must try to reduce the number of nodes communicate directly with the BS, while the optimal number of cluster head $\mathrm{H}$ and wireless energy parameters of the model, $E_{\text {elec }} 、 \varepsilon_{\text {amp }}$ also has a relationship.

Wireless sensor network node layout is completed, the position and energy parameters BS also are finished, the value calculated in advance the number of $\mathrm{H}$ cluster to determine the cluster number, during the lifetime of the whole network cluster number will not change, the sensor network overall energy consumption to a minimum as much as possible, so that it can effectively extend the life cycle of the entire network.

\subsection{Routing algorithm design}

Cluster head is dynamic selection, with maximum residual energy level of the node will become a cluster head, each cluster of regions within the region independently choose the corresponding cluster head, don't need BS centralized control, also do not need additional consultation with other cluster head nodes, so as to ensure the high energy nodes to bear the high energy consumption of the communication business. The routing algorithm of the improved protocol is run 
by round (round), and each wheel is divided into initialization phase and stable operation stage. In the stage of steady work, cluster members according to the assigned TDMA time slot will send data to the cluster head, the cluster head will be fused data forwarding to the local routing tree parent node, until the root node and the root node will transmit data to the BS. After a round of data collection, the new cluster head will be selected, so repeated two stages of work. In order to reduce the overhead of the system, the duration of the stable operation stage should be longer than the initial stage.

After the end of the cluster head selection, the cluster head to the members of the TDMA slot allocation, and establish a route tree for the cluster heads. Cluster head $\mathrm{i}$ broadcast its own information to an adjacent cluster in their own state. Information includes cluster head ID and the current residual energy and its distance from to the BS. Received this message to other cluster head $\mathrm{j}$ based on the received signal strength to calculate the approximate distance from the cluster head ditoj, and the cluster head as his neighbor cluster head temporarily stored. To prepare for the formation for the route tree.

Improved protocol routing algorithm initialization in the first round is under centralized control BS completed. First round of the cluster head to determine routing tree formed after the completion of the first round based routing table, from the second round, because the area has been divided into clusters class and fixed in the first round, the algorithm in the initial stage of the work will be completed only establish cluster head selection and routing tree. In the initialization phase, the local node based solely on its own information to determine the state, no longer need any communication with the BS. In the stable phase, the root node of the tree is responsible for routing data transmitted to the BS, it does not need to communicate with other nodes in the same BS. This will minimize the amount of communication with the BS, thereby greatly reducing the system's energy consumption and prolong the network life cycle.

Cluster head selection, the new cluster head will be the number of nodes within a cluster according to a communication slot allocated to each node within a cluster. Cluster head broadcasts to all nodes in the cluster based on the time slot time division multiplexing manner to inform the node when it can transmit data. After the formation of the cluster head and routing tree, the node can begin transmitting the data. Node continuous collection of information around the cluster, and in their way of communication slots in a single hop communication to transmit data to the corresponding cluster head. Non-cluster head node transmitter can not belong to the node in the communication time slot to close, thereby reducing the energy consumption of nodes. Cluster head must keep their receivers time is turned on, in order to receive all of the data within the cluster nodes transmitted. 


\section{Experiment and Simulation}

Improved protocol algorithm on a simulation platform NS3, compared with $\mathrm{LEACH}$ and $\mathrm{LEACH}-\mathrm{C}$ routing algorithm. Parameter simulation is set in file wireless.tcl and uamp.tcl etc. Details of the parameters is: network coverage area is $200 \mathrm{~m} * 200 \mathrm{~m}$, focal point position $(100-300) \mathrm{m}$, the number of nodes is 200 , the initial energy of sensor nodes is $2 \mathrm{~J}$, Eelec value is $50 \mathrm{~nJ} / \mathrm{bit}$, Ethreshold value is $0.01 \mathrm{~J}$, Eda is the value of $5 \mathrm{~nJ} / \mathrm{bit} / \mathrm{signal}$, packet length is $4000 \mathrm{bits},{ }^{\mathcal{E}_{a m p}}$ is $100 \mathrm{pJ} / \mathrm{bit} / \mathrm{m} 2$.

Since the energy consumption of the cluster head is the most important part of the energy consumption of network simulation comparison chart shows that with the increase of the network coverage area, and increase the distance of the base station node, the cluster head consumption improved protocol is the smallest fluctuations as shown in Fig 2.

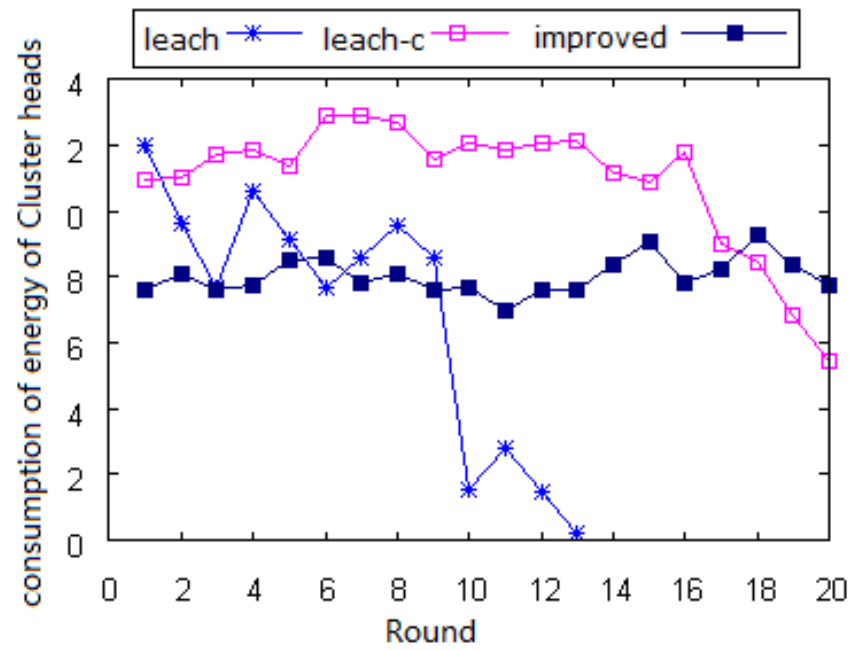

Fig.2. The balance of cluster head energy under the three protocols

Improved routing algorithms FND (First Node Dies) and LND (Last Node Dies) time are LEACH 2 times and 2.5 times. Improved protocol advantage of the routing algorithm is further reflected, this is because in the big scene environment, LEACH algorithm for each round must be re-built cluster, and cluster head to a lot of news broadcasting, the uneven distribution of cluster head position, leading to internal cluster communications also will increase energy consumption. Improved routing algorithm density divided and fixed mechanism cluster class area, which can form a more optimal cluster class structure, communication between nodes is limited within a certain range, thereby reducing the cluster 
internal communications class power consumption. Meanwhile, the routing algorithm reduces the BS communicate directly with the number of nodes.

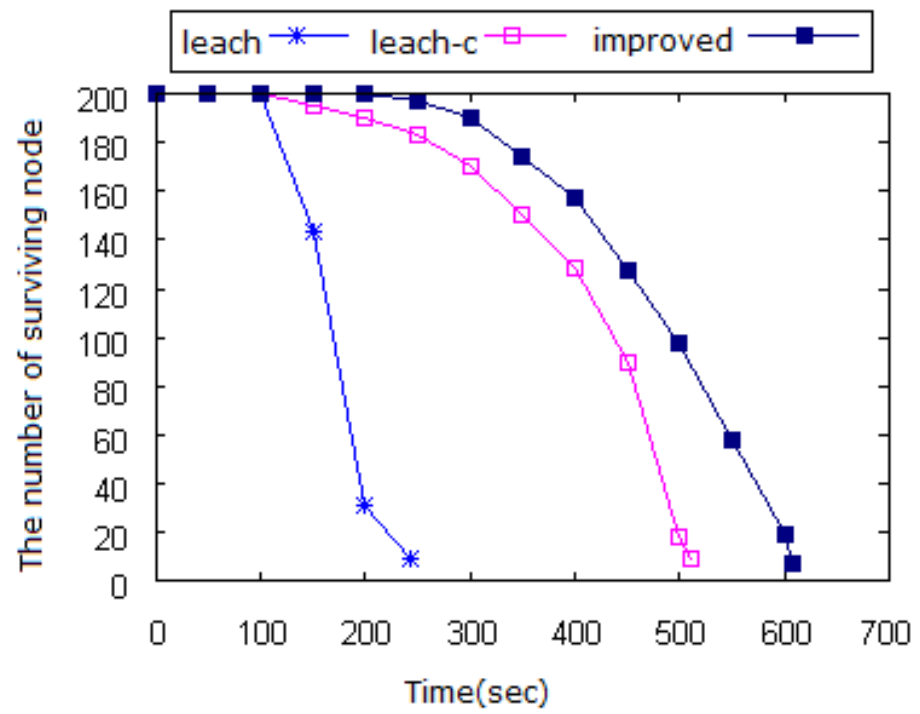

Fig.3.Comparison of the number of surviving nodes in three protocols

Improved algorithm in the entire network energy consumption rate is about 0.51 times the LEACH algorithm, showing that with the expansion of the network size, the improved algorithm energy efficiency of the whole network will be more significant than the LEACH protocol.

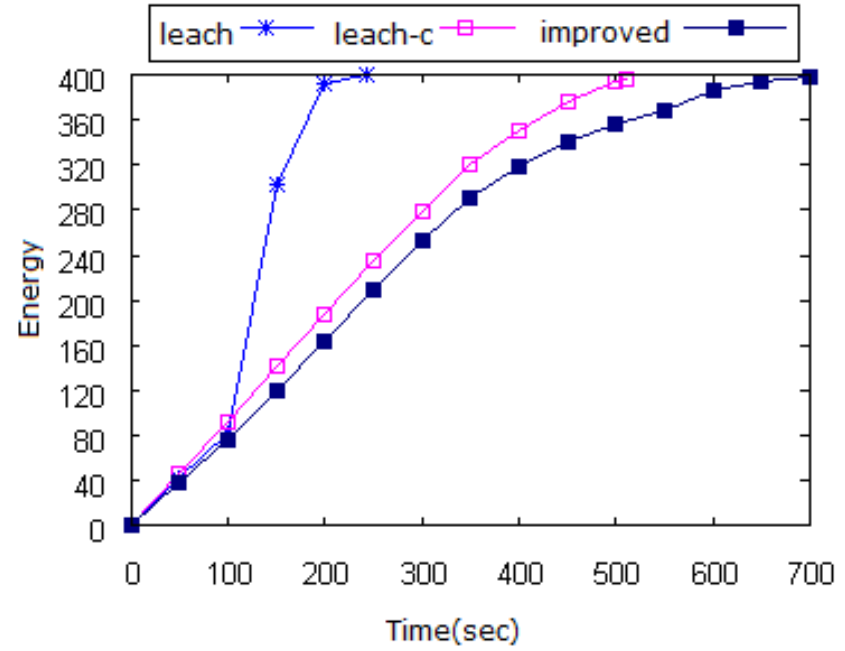

Fig.4. Comparison of total network energy consumption of protocols 


\section{Conclusions}

Based on the density of multi hop routing protocol, through simulation experiments, compared with the Leach protocol and Leach-c protocol. The results show that the improved algorithm can balance the load of the sensor network, save the network energy consumption and prolong the network life cycle. By comparing the simulation results, the algorithm is superior to LEACH in prolonging the network lifetime and improving the accuracy of data. At the same time, with the expansion of the network size, the density based multi hop routing protocol will show better performance.

\section{Acknowledgement}

The Science Foundation of Education Department of Hubei Provincial. (No.B2015199, No.B2015291). The education science planning project of Hubei Province under Grant No.2015GB156.

\section{References}

[1]. WANG Shu-guang.Region Partition Centorid localization Algorithm forWSNs. Transducer and Microsystem Technologies. 2014(12) 149-152

[2]. Mhater V, Rosenberg C. Homogeneous vs heterogeneous clustered sensor networks: a comparative study . IEEE Trans on Mobile Computing, 2004, 6(24) 3646 3651

[3]. O Yonis, et al. HEED: A Hybrid, energy-efficient, distributed clustering approach for ad-hoc sensor networks .IEEE Trans on Mobile Computing, 2004.3(4) 366 379

[4]. SUN Yanjing,LIN Changlin. An Energy Efficient Distributed Uneven Clustering Routing Algorithm for WSNs . Chinese Journal of Sensors and Actuators. 2015(08) 1194-1200.

[5]. W.R.Heinzelman,A.Chandrakasan,H.Balakrishnan. An application-specific Protocol Arehitecture for wireless microsensor networks . IEEE Transaction On Wireless Communications, 2002,1(4) 660 670

[6]. JIANG Hua,LIU Wei-qiang,WANG Xin.Rearch and Improvement of Leach-c Routing Protocol in Wireless Sersor Network .Microelectronics and Computer. 2014(12) 43-47.

[7]. SUN Yanjing;LIN Changlin;JIANG Haifeng. An Energy Efficient Distributed Uneven Clustering Routing Algorithm for WSNs . Chinese Journal of Sensors and Actuators. 2015(08) 1194-1200. 